Proc. of 12th International Workshop on Positron and Positronium Chemistry, August 28-September 1, 2017, Lublin, Poland

\title{
The Elastic Collision of Two H-Like Atoms Including the Exotic Ps and Mu
}

\author{
H. RAY ${ }^{a, b, c, *}$ \\ ${ }^{a}$ Study Center JnganSikha, S-1/407/6, B.P. Township, Kolkata 700094, India \\ ${ }^{b}$ Physics Department, New Alipore College, Kolkata 700053, India \\ ${ }^{c}$ Science Department, National Institute of TTT and Research, Kolkata 700107, India
}

\begin{abstract}
Elastic collision between two H-like atoms using the ab initio static-exchange model (SEM) and a modified static-exchange model (MSEM) at cold energies are investigated in the center of mass frame considering the system as a four-body Coulomb problem where all the Coulomb interaction terms in the direct and exchange channels are treated exactly. The importance of an exact calculation to find basic physics is highlighted. In addition, the dependence of scattering length on the van der Waals interaction between the atoms and the dependence of scattering length on reduced-mass of the system are derived which is completely new information in the field of science.
\end{abstract}

DOI: $10.12693 /$ APhysPolA.132.1473

PACS/topics: 36.10.Dr, 03.65.Nk, 34.50.Cx

\section{Introduction}

Very recently Ray [1-5] introduced two new codes to study cold-atomic collision physics following a SEM and a MSEM. The SEM includes the non-adiabatic short-range effect due to electron exchange. The MSEM includes the effect of long-range van der Waals interaction in addition to the short-range non-adiabatic effect. Both these effects, the non-adiabatic short-range due to electron exchange and the long-range due to induced dipole polarizabilities between the atoms, are highly important at low and cold energies to determine the strength of effective interatomic potential and the scattering length.

The two-atomic system is treated as a four-body Coulomb problem in the center of mass frame and all the Coulomb interaction terms between the atoms were calculated exactly to solve the Schrödinger equation, to obtain the elastic scattering amplitudes. Here the atomic wave functions are exactly known in all H-like systems. The SEM code is applied to study the $\mathrm{Ps}_{\mathrm{s}}-\mathrm{H}, \mathrm{Ps}_{\mathrm{s}}-\mathrm{Ps}_{\mathrm{s}}, \mathrm{Ps}_{\mathrm{s}}-$ $\mathrm{Mu}, \mathrm{Ps}-\mathrm{D}, \mathrm{Ps}-\mathrm{T}, \mathrm{Mu}-\mathrm{Mu}, \mathrm{Mu}-\mathrm{H}, \mathrm{Mu}-\mathrm{D}, \mathrm{Mu}-\mathrm{T}, \mathrm{H}-\mathrm{H}$, $\mathrm{H}-\mathrm{D}, \mathrm{H}-\mathrm{T}, \mathrm{D}-\mathrm{D}, \mathrm{D}-\mathrm{T}$ and $\mathrm{T}-\mathrm{T}$ systems. Here Ps, Mu, $\mathrm{H}, \mathrm{D}, \mathrm{T}$ symbolize the positronium, muonium, hydrogen, deuterium, and tritium, respectively. The MSEM code is used to study the variation of scattering length with the variation of the strength of attractive long-range van der Waals interaction controlling the minimum interatomic distances $\left(R_{0}\right)$ as $2 a_{0}, 3 a_{0}, 4 a_{0}, 5 a_{0}, 6 a_{0}, 7 a_{0}$, $8 a_{0}, 9 a_{0}, 10 a_{0}, 11 a_{0}, 12 a_{0}, 15 a_{0}, 20 a_{0}$. Here $a_{0}$ indicates the Bohr radius. The systems studied are $\mathrm{Ps}_{\mathrm{s}}-\mathrm{H}$ and $\mathrm{H}-$ H. There is no cold energy experimental data available to compare. So the derived scattering lengths for $\mathrm{H}-\mathrm{H}$

*e-mail: hasi_ray@yahoo.com and $\mathrm{Ps}_{\mathrm{S}}-\mathrm{H}$ systems are compared with available theoretical data and found in agreement. The present code is fit to calculate the low and cold energy data. If the incident momentum $k$ is greater than 10 a.u., convergence problem starts in all the systems. The incident energy $E[\mathrm{eV}]$ is related with $k$ [a.u.] by the relation $E=27.21 k^{2} / 2 \mu$, where $\mu$ is the reduced mass of the system in a.u. Both the codes are investigated thoroughly and reproduce exactly the same data and establish firmly the earlier findings.

\section{Theory and results}

An example of a H-like two-atomic system is presented in Fig. 1. The total Hamiltonian of the system is

$$
\begin{aligned}
H & =H_{\text {Direct }}=-\frac{\hbar^{2}}{2 \mu} \nabla_{R^{\prime}}^{2}+H_{A}\left(\boldsymbol{r}_{1 \mathrm{~A}}\right)+H_{B}\left(\boldsymbol{r}_{2 \mathrm{~B}}\right) \\
& +V_{\text {Direct }}\left(\boldsymbol{r}_{1 \mathrm{~A}}, \boldsymbol{r}_{2 \mathrm{~B}}, \boldsymbol{R}\right)=H_{\text {Exchange }}=-\frac{\hbar^{2}}{2 \mu} \nabla_{R_{f}}^{2} \\
& +H_{A}\left(\boldsymbol{r}_{2 \mathrm{~A}}\right)+H_{B}\left(\boldsymbol{r}_{1 \mathrm{~B}}\right)+V_{\text {Exchange }}\left(\boldsymbol{r}_{1 \mathrm{~A}}, \boldsymbol{r}_{2 \mathrm{~B}}, \boldsymbol{R}\right) .
\end{aligned}
$$

Here $\mu$ is the reduced mass of the system, $H_{A}$ and $H_{B}$ are

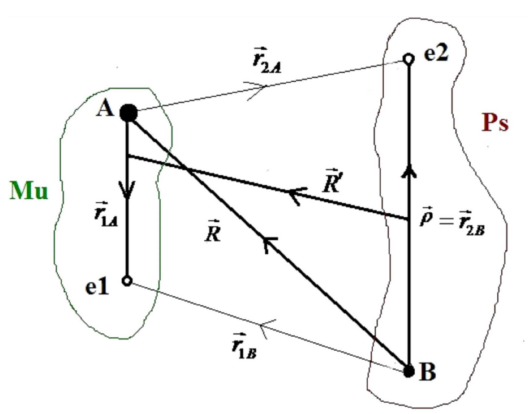

Fig. 1. The Mu $1 s-\mathrm{Ps}(1 s)$ system. 
the Hamiltonians of the two atoms and are defined as:

$$
\begin{aligned}
& H_{A}\left(\boldsymbol{r}_{1 \mathrm{~A}}\right)=-\frac{\hbar^{2}}{2 \mu_{A}} \nabla_{r_{1 A}}^{2}-\frac{1}{\left|\boldsymbol{r}_{1 \mathrm{~A}}\right|}, \\
& H_{B}\left(\boldsymbol{r}_{2 \mathrm{~B}}\right)=-\frac{\hbar^{2}}{2 \mu_{B}} \nabla_{r_{2 B}}^{2}-\frac{1}{\left|\boldsymbol{r}_{2 \mathrm{~B}}\right|},
\end{aligned}
$$

$V\left(\boldsymbol{R}, \boldsymbol{r}_{1 \mathrm{~A}}, \boldsymbol{r}_{2 \mathrm{~B}}\right)$ is the Coulomb interaction: $V_{\text {Direct }}$ is for the direct channel and $V_{\text {Exchange }}$ is for the exchange or rearrangement channel

$$
\begin{aligned}
& V_{\text {Direct }}\left(\boldsymbol{R}, \boldsymbol{r}_{1 \mathrm{~A}}, \boldsymbol{r}_{2 \mathrm{~B}}\right)=\frac{Z_{A} Z_{B}}{R}-\frac{Z_{A}}{\left|\boldsymbol{R}-\boldsymbol{r}_{2 \mathrm{~B}}\right|} \\
& -\frac{Z_{B}}{\left|\boldsymbol{R}+\boldsymbol{r}_{1 \mathrm{~A}}\right|}+\frac{1}{\left|\boldsymbol{R}+\boldsymbol{r}_{1 \mathrm{~A}}-\boldsymbol{r}_{2 \mathrm{~B}}\right|}, \\
& V_{\text {Exchange }}\left(\boldsymbol{R}, \boldsymbol{r}_{1 \mathrm{~A}}, \boldsymbol{r}_{2 \mathrm{~B}}\right)=\frac{Z_{A} Z_{B}}{R}-\frac{Z_{A}}{\left|\boldsymbol{r}_{1 \mathrm{~A}}\right|} \\
& -\frac{Z_{B}}{\left|\boldsymbol{r}_{2 \mathrm{~B}}\right|}+\frac{1}{\left|\boldsymbol{R}+\boldsymbol{r}_{1 \mathrm{~A}}-\boldsymbol{r}_{2 \mathrm{~B}}\right|} .
\end{aligned}
$$

The four Coulomb interaction terms in Eqs. (3a) and $(3 \mathrm{~b})$ : the first one is the nucleus-nucleus (NN) interaction, the fourth one is the electron-electron $\left(\mathrm{e}_{1} \mathrm{e}_{2}\right)$ interaction, the second one is the interaction between nucleus $\mathrm{A}$ and electron $2(\mathrm{Ae})$, and the third one is the interaction between nucleus $\mathrm{B}$ and electron $1(\mathrm{Be})$.

The system wave functions for the initial and final channels are defined as:

$$
\begin{aligned}
& \psi_{i}=\mathrm{e}^{\mathrm{i} \boldsymbol{k}_{i} \boldsymbol{R}^{\prime}} \varphi_{1 s}^{A}\left(\boldsymbol{r}_{1 \mathrm{~A}}\right) \eta_{1 s}^{B}\left(\boldsymbol{r}_{2 \mathrm{~B}}\right), \\
& \psi_{f}=\left(1 \pm P_{12}\right) \mathrm{e}^{\mathrm{i} \boldsymbol{k}_{f} \boldsymbol{R}_{f}} \varphi_{1 s}^{A}\left(\boldsymbol{r}_{1 \mathrm{~A}}\right) \eta_{1 s}^{B}\left(\boldsymbol{r}_{2 \mathrm{~B}}\right) .
\end{aligned}
$$

Here $\varphi_{1 s}^{A}\left(\boldsymbol{r}_{1 \mathrm{~A}}\right)$ and $\eta_{1 s}^{B}\left(\boldsymbol{r}_{2 \mathrm{~B}}\right)$ are the ground state wave functions of the atoms and $P_{12}$ is the exchange (or antisymmetry) operator. The vector joining the center of masses of the two atoms in the direct channel is

$$
\boldsymbol{R}^{\prime}=\boldsymbol{R}+\frac{m_{\mathrm{e}}}{m_{\mathrm{A}}+m_{\mathrm{e}}} \boldsymbol{r}_{1 \mathrm{~A}}-\frac{m_{\mathrm{e}}}{m_{\mathrm{B}}+m_{\mathrm{e}}} \boldsymbol{r}_{2 \mathrm{~B}},
$$

whereas the same in the exchange channel is

$$
\begin{gathered}
\boldsymbol{R}_{f}=\boldsymbol{R}+\frac{m_{\mathrm{e}}}{m_{\mathrm{A}}+m_{\mathrm{e}}}\left(\boldsymbol{r}_{2 \mathrm{~B}}-\boldsymbol{R}\right) \\
-\frac{m_{\mathrm{e}}}{m_{\mathrm{B}}+m_{\mathrm{e}}}\left(\boldsymbol{r}_{1 \mathrm{~A}}+\boldsymbol{R}\right) .
\end{gathered}
$$

The notations $m_{\mathrm{A}}, m_{\mathrm{B}}, m_{\mathrm{e}}$ represent the masses of the nucleus A, nucleus B and the mass of electron. The $\boldsymbol{k}_{i}$ and $\boldsymbol{k}_{f}$ represent the initial and final momenta of the projectile. In elastic scattering $\left|\boldsymbol{k}_{i}\right|=\left|\boldsymbol{k}_{f}\right|$, so only the direction of the final momentum $\boldsymbol{k}_{f}$ changes. Here $\boldsymbol{R}$ is the inter-nucleus displacement, $\boldsymbol{r}_{1 \mathrm{~A}}$ and $\boldsymbol{r}_{2 \mathrm{~B}}$ are the position vectors of the two system electrons with respect to their corresponding atomic nuclei. The atomic unit (a.u.) is used throughout.

Projecting different states on the Schrödinger equation just like the Hartree-Fock variational method one can get the integro-differential equations that can be solved by the method of iteration. Here the Lippman-Schwinger type integral equation in the momentum space formalism [6] rather than using the coordinate space adapted by Fraser in Ref. [7] is used. The formally exact LippmanSchwinger type coupled integral equation for the scattering amplitude in momentum space is given by [6]:

$$
\begin{aligned}
& f_{n^{\prime} 1 s, n 1 s}^{ \pm}\left(\boldsymbol{k}_{f}, \boldsymbol{k}_{i}\right)=B_{n^{\prime} 1 s, n 1 s}^{ \pm}\left(\boldsymbol{k}_{f}, \boldsymbol{k}_{i}\right) \\
& \quad-\sum_{n^{\prime \prime}} \int \frac{\mathrm{d} \boldsymbol{k}^{\prime \prime}}{2 \pi^{2}} \frac{B_{n^{\prime} 1 s, n^{\prime \prime} 1 s}^{ \pm}\left(\boldsymbol{k}_{f}, \boldsymbol{k}^{\prime \prime}\right) f_{n^{\prime \prime} 1 s, n 1 s}^{ \pm}\left(\boldsymbol{k}^{\prime \prime}, \boldsymbol{k}_{i}\right)}{\boldsymbol{k}_{n^{\prime \prime} 1 s}^{2}-\boldsymbol{k}^{\prime \prime 2}+\mathrm{i} \varepsilon} .
\end{aligned}
$$

Here $B^{ \pm}$are the well known Born-Oppenheimer (BO) scattering amplitude [1, 8-11] in the singlet $(+)$ and triplet $(-)$ channels, respectively. In a similar fashion, $f^{ \pm}$ indicate the unknown scattering amplitudes for the singlet and triplet states of the two system electrons. Generally, the partial wave analysis is used to reduce the threedimensional integral equation into the one-dimensional form. Here the BO amplitude $\left(B^{ \pm}\right)$acts as the input to get the SEM amplitude following Eq. (6) and is defined as:

$$
\begin{aligned}
& B_{n^{\prime} 1 s, n 1 s}^{ \pm}\left(\boldsymbol{k}_{f}, \boldsymbol{k}_{i}\right)=-\frac{\mu}{2 \pi} \int \mathrm{d} \boldsymbol{R} \mathrm{d} \boldsymbol{r}_{1 \mathrm{~A}} \mathrm{~d} \boldsymbol{r}_{2 \mathrm{~B}} \\
& \quad \times \psi_{f}^{*}\left(\boldsymbol{R}, \boldsymbol{r}_{1 \mathrm{~A}}, \boldsymbol{r}_{2 \mathrm{~B}}\right) V\left(\boldsymbol{R}, \boldsymbol{r}_{1 \mathrm{~A}}, \boldsymbol{r}_{2 \mathrm{~B}}\right) \psi_{i}\left(\boldsymbol{R}, \boldsymbol{r}_{1 \mathrm{~A}}, \boldsymbol{r}_{2 \mathrm{~B}}\right) .
\end{aligned}
$$

The MSEM theory is introduced to include the effect of van der Waals interaction. The definition of van der Waals interaction used by Barker and Bransden [12] is used as described below

$$
\begin{aligned}
& V_{\text {van }}(R)=0, \text { if } R<R_{0}, \\
& V_{\text {van }}(R)=-\frac{C_{W}}{R^{6}}, \text { if } R \geq R_{0} \text { when } R_{0} \rightarrow 0 .
\end{aligned}
$$

The corresponding potential is defined as:

$$
\begin{aligned}
& B_{v a n}-\int \mathrm{d} \boldsymbol{r}_{1 \mathrm{~A}} \int \mathrm{d} \boldsymbol{r}_{2 \mathrm{~B}} \int \mathrm{d} \hat{R} \int_{R=R_{0}}^{\infty} \mathrm{d} R R^{2} \\
& \times\left(\psi^{*}\left(\boldsymbol{R}, \boldsymbol{r}_{1 \mathrm{~A}}, \boldsymbol{r}_{2 \mathrm{~B}}\right) \frac{C_{W}}{R^{6}} \psi\left(\boldsymbol{R}, \boldsymbol{r}_{1 \mathrm{~A}}, \boldsymbol{r}_{2 \mathrm{~B}}\right)\right) .
\end{aligned}
$$

Here $C_{W}$ is replaced by van der Waals $C_{6}$ coefficient. How $R_{0}$ will be determined is an important question? In $\mathrm{H}_{2}$ molecule, the internuclear separation is $0.74 \AA$, i.e. 1.4 a.u. [13].

The effective range theory expresses the $s$-wave elastic phase shift $\left(\delta_{0}\right)$ as a function of scattering-length $(a)$ and projectile energy $\left(\sim k^{2}\right)$ so that

$$
k \cot \delta_{0}=-\frac{1}{a}+\frac{1}{2} r_{0} k^{2}+O\left(k^{4}\right),
$$

when $k$ is the magnitude of the incident momentum and $r_{0}$ is the range of the potential.

In Fig. 2, the $s$-wave elastic phase shifts of both the total spin aligned states: $S=0$ for the singlet $(+)$ and $S=2$ for the triplet $(-)$ for $\operatorname{Ps}(1 s)-\operatorname{Ps}(1 s)$ collision using SEM are presented against the incident momenta $k=0.1$ to $k=0.6$ a.u. in the energy region below the threshold. These data are compared with the data of Ivanov et al. [14].

The effective range theory is useful to calculate the scattering length. In Table I, the scattering length data for different Ps-atomic systems are presented. A dependence of scattering length on the reduced-mass of the system is observed. The present findings could be useful to explain the electron-like scattering behavior of Ps [15] since the reduced-mass of $\mathrm{Ps}_{\mathrm{S}-\text { atom }}$ and electron-atom 


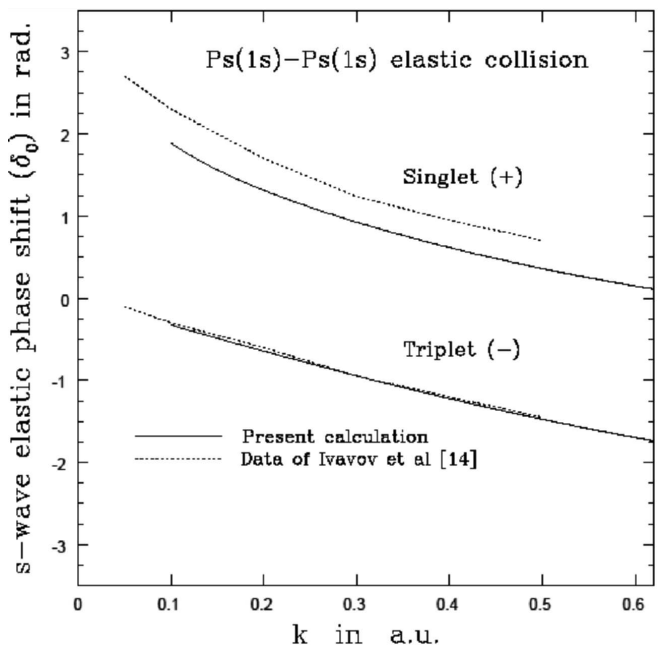

Fig. 2. The $s$-wave elastic phase shifts in radian for both singlet $(+)$ and triplet $(-)$ channels in $\operatorname{Ps}(1 s)_{-}^{-}$ $\operatorname{Ps}(1 s)$ scattering against the incident momentum $k$ in a.u., using SEM..

TABLE I

The variation of singlet $(+)$ and triplet $(-)$ scattering length $\lambda$ [a.u.] with reduced mass $\alpha$ [a.u.] of the systems: Ps-Ps, Ps-Mu, Ps-H, Ps-D and Ps-T using SEM.

\begin{tabular}{|c|c|c|c|c|c|}
\hline System & Ps-Ps & $\mathrm{Ps}-\mathrm{Mu}$ & $\mathrm{Ps}-\mathrm{H}$ & $\mathrm{Ps}_{\mathrm{S}}-\mathrm{D}$ & $\mathrm{Ps}_{\mathrm{S}}-\mathrm{T}$ \\
\hline$\alpha$ & 1.0 & 1.9809 & 1.9978 & 1.9989 & 1.9993 \\
\hline$(+)$ & 9.35 & 7.40 & 7.24 & 7.18 & 7.14 \\
\hline$(-)$ & 3.25 & 2.50 & 2.48 & 2.46 & 2.45 \\
\hline
\end{tabular}

systems are almost the same and the difference decreases as the atom becomes heavier.

Figure 3 shows the plot of the effective range theory used to calculate the scattering lengths for heavier systems. The variation of triplet scattering length with

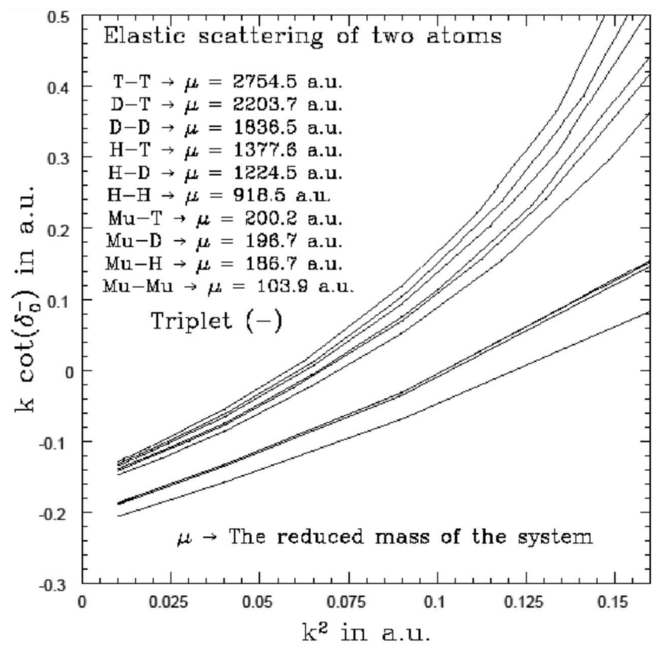

Fig. 3. $\quad k \cot \delta_{0}^{-}$vs. $k^{2}$ curve for different H-like systems using SEM. The reduced masses are increasing gradually for the systems from bottom to top solid curves.

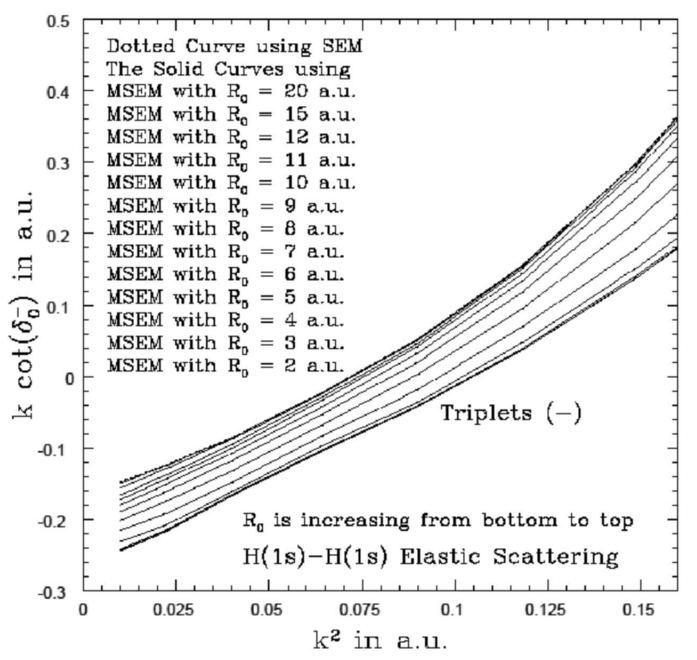

Fig. 4. $\quad k \cot \delta_{0}^{-}$vs. $\quad k^{2}$ plot for $\mathrm{H}(1 s)-\mathrm{H}(1 s)$ elastic scattering using SEM and MSEM for $R_{0}=2 a_{0}, 3 a_{0}$, $4 a_{0}, 5 a_{0}, 6 a_{0}, 7 a_{0}, 8 a_{0}, 9 a_{0}, 10 a_{0}, 11 a_{0}, 12 a_{0}, 15 a_{0}$, $20 a_{0}$.

reduced-mass for the heavier atomic systems e.g. $\mathrm{Mu}-$ $\mathrm{Mu}, \mathrm{Mu}-\mathrm{H}, \mathrm{Mu}-\mathrm{D}, \mathrm{Mu}-\mathrm{T}, \mathrm{H}-\mathrm{H}, \mathrm{H}-\mathrm{D}, \mathrm{H}-\mathrm{T}, \mathrm{D}-\mathrm{D}, \mathrm{D}-$ $\mathrm{T}, \mathrm{T}-\mathrm{T}$ are presented in Table II. Again we are finding a systematic dependence of scattering length with the reduced-mass of the system [5]. It should be noted that the SEM theory include only the non-adiabatic shortrange effects due to exchange, but no long-range interaction.

TABLE II

The variation of triplet scattering length $\lambda$ [a.u.] with reduced mass $\alpha$ [a.u.] of different H-like systems.

\begin{tabular}{c|c|c|c|c|c|c|c|c|c|c}
\hline \hline & Mu-Mu & Mu-H & Mu-D & Mu-T & H-H & H-D & H-T & D-D & D-T & T-T \\
\hline$\alpha$ & 103.9 & 186.7 & 196.7 & 200.2 & 918.5 & 1224.5 & 1377.6 & 1836.5 & 2203.7 & 2754.5 \\
$\lambda$ & 4.54 & 4.76 & 4.88 & 4.95 & 5.88 & 6.25 & 6.37 & 6.58 & 6.68 & 6.90
\end{tabular}

The MSEM code is used to study the effect of longrange attractive van der Waals interaction in (i) Ps $1 s^{-}$ $\mathrm{H} 1 s$ and (ii) $\mathrm{H} 1 s-\mathrm{H} 1 s$ systems to study the elastic scattering. The most accurate values for the van der Waals $C_{6}$ coefficients are used [22] to define the long-range potentials in Eq (8). Different minimum values of interatomic distance $R_{0}$ are chosen to vary the strength of interatomic potential. The variation of scattering length with the variation of values of $R_{0}$ for Ps-H system are tabulated and compared with SEM data in Table III. It is found that at larger interatomic distances e.g. at $R_{0}=15 a_{0}$, the MSEM and SEM data are almost the same in both the theories. The scattering lengths in both the systems are gradually decreasing with the increase of the strength of attractive van der Waals interaction. The findings are consistent with the basic physics that the stronger attractive potential causes the shorter scattering length and the stronger repulsive potential causes the longer scattering length [5].

In Fig. 4, the plot applying effective range theory for different values of $R_{0}$ is presented for $\mathrm{H}-\mathrm{H}$ system. With the increase of the values of $R_{0}$, the MSEM data are be- 
ing gradually closer to the SEM data. As the value of $R_{0}$ decreases, the strength of attractive van der Waals interaction gradually increases. The variation of scattering length with different values of $R_{0}$ for $\mathrm{H}-\mathrm{H}$ system are presented in Table IV. It is to be observed that the scattering length is gradually decreasing and being closer to the reported accurate values [23-27] as the strength of van der Waals interaction increases with decreasing $R_{0}$. The systematic variation of scattering length towards the accurate values with decrease of $R_{0}$ has a great significance to understand the basic physics of two-atomic systems.

TABLE III

The comparison of $s$-wave elastic scattering length $\lambda$ and effective ranges $\left(r_{0}\right)$ in atomic units for electron spin singlet $(+)$ and triplet $(-)$ in Ps-H system using SEM and MSEM.

\begin{tabular}{rl|c|c|c|c|c|c|c|c}
\hline \hline & SEM & \multicolumn{7}{|c|}{ MSEM data for $R_{0}=$} & Other \\
\cline { 3 - 8 } & data & $10 a_{0}$ & $7 a_{0}$ & $5 a_{0}$ & $4 a_{0}$ & $3 a_{0}$ & $2.5 a_{0}$ & results \\
\hline$\lambda(+)$ & 7.25 & 7.22 & 7.19 & 7.14 & 7.04 & 6.17 & 5.32 & $4.5^{a}, 5.22^{b}, 5.20^{c}, 5.84^{d}, 3.49^{e}, 4.30^{f}$ \\
$r_{0}(+)$ & 3.79 & 3.78 & 3.77 & 3.75 & 3.74 & 3.73 & 3.48 & $2.2^{a}, 2.90^{d}, 2.08^{f}$ \\
$\lambda(-)$ & 2.49 & 2.45 & 2.43 & 2.36 & 2.27 & 2.12 & 2.02 & $2.36^{a}, 2.41^{b}, 2.45^{c}, 2.32^{d}, 2.46^{e}, 2.2^{f}$ \\
$r_{0}(-)$ & 1.42 & 1.41 & 1.40 & 1.38 & 1.34 & 1.28 & 1.19 & $1.31^{a}$ \\
\\
${ }^{a}$ stabilization calculation of Drachman and Houston [16], ${ }^{b}$ close-coupling calculation of Sinha et al. [17], \\
${ }^{c}$ R-matrix calculation of Blackwood et al. [18], ${ }^{d}$ Kohn variational calculation of Page [19], ${ }^{e}$ variational calcu- \\
lation of Adhikari and Mandal [20], ${ }^{f}$ stochastic variational calculation of Ivanov et al. [21].
\end{tabular}

TABLE IV

The scattering length $\lambda$ [a.u.] using for different values of $R_{0}$ for $\mathrm{H}-\mathrm{H}$ system using SEM and MSEM.

\begin{tabular}{|c|c|c|c|c|c|c|c|c|c|c|c|c|c|c|}
\hline \multirow{2}{*}{$\begin{array}{l}\text { SEM } \\
\text { data }\end{array}$} & \multicolumn{13}{|c|}{ MSEM with $R_{0}=$} & \multirow{2}{*}{$\begin{array}{c}\text { Other } \\
\text { data }\end{array}$} \\
\hline & $20 a_{0}$ & $15 a_{0}$ & $12 a_{0}$ & $11 a_{0}$ & $10 a_{0}$ & $9 a_{0}$ & $8 a_{0}$ & $7 a_{0}$ & $6 a_{0}$ & $5 a_{0}$ & $4 a_{0}$ & $3 a_{0}$ & $2 a_{0}$ & \\
\hline $5.88,5.90^{a}$ & 5.80 & 5.68 & 5.26 & 5.11 & 4.89 & 4.63 & 4.38 & 4.03 & 3.77 & 3.68 & 3.63 & 3.60 & 3.58 & $2.04^{a} 1.91^{b}, 1.22^{c}, 1.34^{d}, 1.3^{e}$ \\
\hline
\end{tabular}

Baird [27].

All the results in summary form are presented in Fig. 5 and in Fig. 6. Figure 5 presents the interesting behavior of scattering length with the variation of the reduced masses of the systems. Figure 6 describes the interesting behavior of scattering lengths with the variation of the strength of van der Waals interaction for $\mathrm{H}-\mathrm{H}$ triplet, $\mathrm{Ps}_{\mathrm{s}} \mathrm{H}$ singlet and $\mathrm{Ps}_{\mathrm{s}}-\mathrm{H}$ triplet.

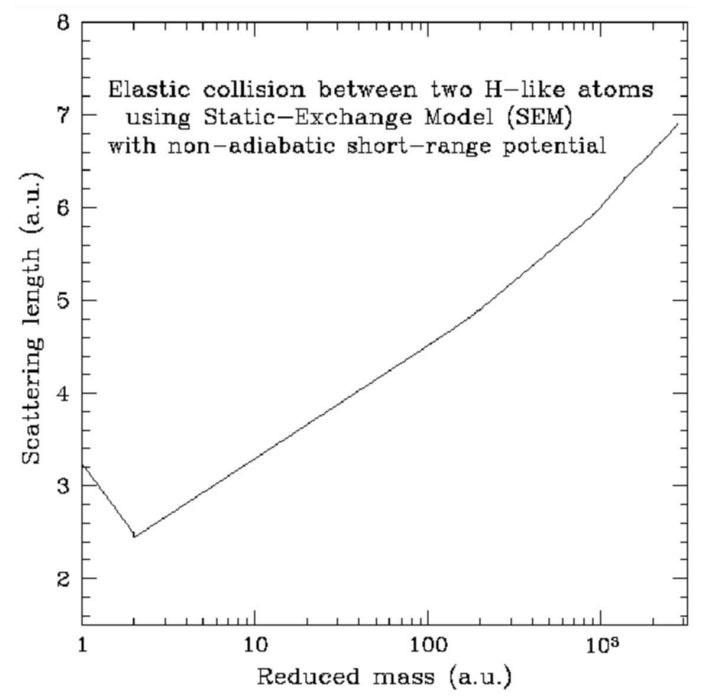

Fig. 5. The behavior of scattering length with reduced mass of the system using SEM.

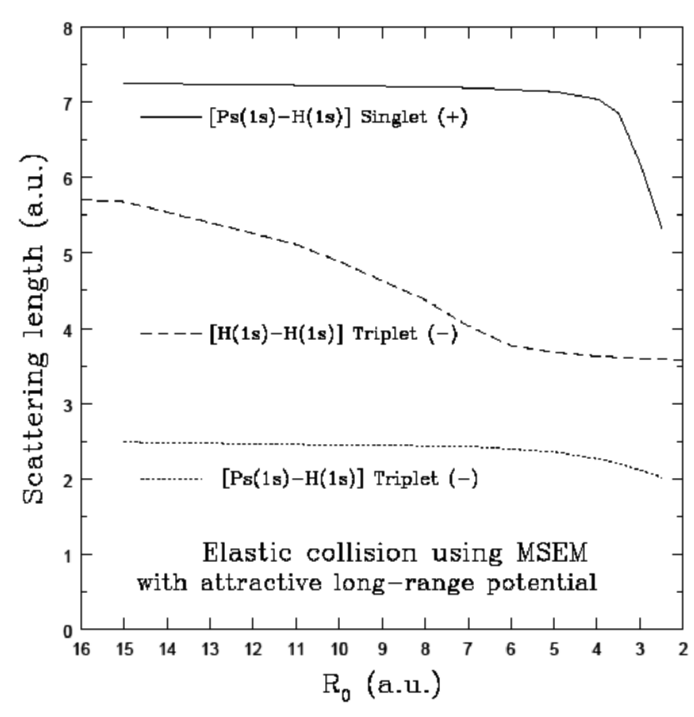

Fig. 6. The behavior of scattering length with the strength of van der Waals interaction using MSEM.

In summary the present article I have presented a brief description of the two-atomic collision phenomena. The studies that are done here are just the starting works in the area. By a little modification, one could modify the codes to apply them in the alkali atomic systems. To include almost the entire non-adiabatic effect, one has to consider the first excited $s$-states of both the atoms in coupled-channel methodology. As reduced-mass 
of the system is an important parameter to determine the strength of interatomic potential, we could answer a few unanswered questions e.g. electron-like behavior of $\mathrm{Ps}_{\mathrm{s}}$ [15]. The most accurate values of $C_{6}$ coefficients for all the combinations of two-atomic systems would be useful to answer the reason of the Bose-Einstein condensation (BEC) formation in $\mathrm{Rb}^{85}-\mathrm{Rb}^{85}$ system. It is to be noted that the reduced-mass of $\mathrm{Rb}^{85}-\mathrm{Rb}^{85}$ system is 78048.5 a.u. In $\mathrm{Rb}^{85}-\mathrm{Rb}^{87}$ system it is 78955.8 a.u. and in $\mathrm{Rb}^{87}-\mathrm{Rb}^{87}$ system it is 79884.5 a.u. Both are much higher than the $\mathrm{Rb}^{85}-\mathrm{Rb}^{85}$ system.

\section{Acknowledgments}

The author is thankful to DST, Govt. of India for Grant No. SR/WOSA/PS-13/2009.

\section{References}

[1] H. Ray, Pramana 83, 914 (2014).

[2] H. Ray, Pramana 86, 1077 (2016).

[3] H. Ray, Pramana 87, 8 (2016).

[4] H. Ray, J. Phys. Conf. Ser. 618, 012008 (2015).

[5] H. Ray, in: POSMOL2015, Book of Abstract, 2015, p. 52 .

[6] A.S. Ghosh, N.C. Sil, P. Mondal, Phys. Rep. 87, 313 (1982).

[7] P.A. Fraser, Proc. R. Soc. B 78, 329 (1961); J. Phys. B 1, 1006 (1968).

[8] H. Ray, A.S. Ghosh, J. Phys. B 29, 5505 (1996); ibid. 30, 3745 (1997); ibid. 31, 4427 (1998).

[9] H. Ray, in: GSFC NASA Conf. Proc. on Atomic and Molecular Physics, Ed. A.K. Bhatia, NASA/CP-2006214146, NASA 2007, p. 121.
[10] H. Ray, J. Phys. B 32, 5681 (1999); ibid. 33, 4285 (2000); ibid. 35, 2625 (2002).

[11] H. Ray, Phys. Rev. A 73, 064501 (2006).

[12] M.I. Barker, B.H. Bransden, J. Phys. B 1, 1109 (1968); ibid. 2, 730 (1969).

[13] W.J. Moore, Physical Chemistry, 2nd ed., PrenticeHall Chemistry Series, Ch. 11, p. 298.

[14] I.A. Ivanov, J. Mitroy, K. Varga, Phys. Rev. A 65, 022704 (2002).

[15] S.J. Brawley, S.E. Fayer, M. Shipman, G. Laricchia, Science 330, 789 (2010).

[16] J. Mitroy, M.W.J. Bromley, Phys. Rev. A 68, 035201 (2003).

[17] R.J. Drachman, S.K. Houston, Phys. Rev. A 12, 885 (1975); ibid. 14, 894 (1976).

[18] P.K. Sinha, A. Basu, A.S. Ghosh, J. Phys. B 33 , 2579 (2000)

[19] J.E. Blackwood, M.T. McAlinden, H.R.J. Walters, Phys. Rev. A 65, (2002).

[20] B.A.P. Page, J. Phys. B 9, 1111 (1976).

[21] S.K. Adhikari, P. Mandal, J. Phys. B 34, L187 (2001).

[22] I.A. Ivanov, J. Mitroy, K. Varga, Phys. Rev. Lett. Phys. Rev. Lett. 87, 063201 (2001).

[23] A. Sen, S. Chakraborty, A.S. Ghosh, Europhys. Lett. 76, $582(2006)$

[24] M.J. Jamieson, A. Dalgarno, J.N. Yukich, Phys. Rev. A 46, 6956 (1992).

[25] M.J. Jamieson, A. Dalgarno, J. Phys. B 31, L219 (1998).

[26] C.J. Williams, P.S. Julienne, Phys. Rev. A 47, 1524 (1995).

[27] N. Koyama, J.C. Baird, J. Phys. Soc. Jpn. 55, 801 (1986). 